J. Microbiol. Biotechnol. (2017), 27(2), 357-364

http://dx.doi.org/10.4014/jmb.1609.09036

\title{
Corrigendum to: Molecular Characterization of Adenylyl Cyclase Complex Proteins Using Versatile Protein-Tagging Plasmid Systems in Cryptococcus neoformans
}

\author{
Yee-Seul So ${ }^{1 \dagger}$, Dong-Hoon Yang ${ }^{1 \dagger}$, Kwang-Woo Jung ${ }^{1}$, Won-Ki Huh ${ }^{2}$, and Yong-Sun Bahn ${ }^{1 *}$ \\ ${ }^{1}$ Department of Biotechnology, Yonsei University, Seoul 03722, Republic of Korea \\ ${ }^{2}$ Department of Biological Sciences, Seoul National University, Seoul 08826, Republic of Korea
}

In the article titled "Molecular Characterization of Adenylyl Cyclase Complex Proteins

Using Versatile Protein-Tagging Plasmid Systems in Cryptococcus neoformans", the authors noticed that the B4028 primer sequence was given incorrectly in the Table. S1. The correct primer sequence is 5'-CGCAAGCTTGGAGCCATGAAGATCCTGA3. The correct 'Table S1' is now available online. Furthermore, we found typos in the supplementary data and revised them as follow. 'Fig. 2. Melanin and capsule analyses of tagging strains' should be changed to 'Fig. S2. Melanin and capsule analyses of tagging strains'. 'Table 2. Strains used in this study' should be changed to 'Table S2. Strains used in this study'. 'Table 2. Plasmid used in this study' should be changed to 'Table S3. Plasmids used in this study'. 